CERN-TH/2000-152

UCLA $/ 00 /$ TEP $/ 18$

October 22, 2018

\title{
CONFORMAL FIELDS IN HIGHER DIMENSIONS *
}

\author{
S. Ferrara ${ }^{\dagger \ddagger}$ and C. Frønsdal ${ }^{\dagger}$ \\ $\dagger$ Physics Department, University of California Los Angeles CA 90095-1547, USA \\ $\ddagger$ CERN Theoretical Division, CH 1211 Geneva 23 Switzerland
}

\begin{abstract}
We generalize, to any space-time dimension, the unitarity bounds of highest weight UIR's of the conformal groups with Lie algebras $s o(2, d)$. We classify gauge theories invariant under $s o(2, d)$, both integral and half-integral spins. A similar analysis is carried out for the algebras $s o^{*}(2 n)$.

We study new unitary modules of the conformal algebra in $d>4$, that have no analogue for $d \leq 4$ as they cannot be obtained by "squaring" singletons. This may suggest the interpretation of higher dimensional non-trivial conformal field theories as theories of "tensionless" $p$-branes of which tensionless strings in $d=6$ are just particular examples.
\end{abstract}

\section{Introduction.}

Extensive work on $A d S_{d+1}$ and its relation to conformal field theories on $\mathcal{M}_{d}^{c}=$ $\partial A d S_{d+1}$ has found an interesting realization in supergravity and string and $M$ theory by relating the horizon geometry of $p$-branes to the world-volume dynamics of the

* To be presented to the International Conference dedicated to the memory of Professor Efim Fradkin, Moscow June 5-10, 2000, and to the Ninth Marcel Grossmann meeting, Rome July 2-8 2000. 
brane $[\mathrm{GiT}]$. The conjectured duality $[\mathrm{Mal}][\mathrm{GKP}][\mathrm{W} 1]$ between theories of gravity and boundary conformal field theories is particularly powerful in the case of space-time supersymmetric field theories where the $p$-brane can be "BPS" saturated; that is, when its world volume dynamics preserves some fraction $\leq N / 2$ of the original $N$ supersymmetries. Theories with maximal supersymmetry correspond to $p=d-1=2,5$ and 3 respectively in $M$ theory and in IIB string theory compactified on $A d S_{d+1} \times S_{D-d-1}$ $[\mathrm{GuT}]$. In these theories a peculiar phenomenon already occurs for $D=11, d=6$ where the world-volume $M$-theory five brane $(2,0)$ six-dimensional field theory is believed to be a non-trivial and interacting theory of "tensionless self-dual strings" [W2][SW][Se][St]. The $A d S_{7} / C F T_{6}$ correspondence [Mal] predicts that such a theory, at least in a certain regime, must be holographically equivalent to $11 \mathrm{D}$ supergravity on $A d S_{7} \times S_{4}$ and in fact certain $\frac{1}{2}$ BPS states of the latter (such as the K-K towers) [GVNW] can be uniquely identified with short representations of the $(2,0)$ superconformal algebra $[\mathrm{AOY}][\mathrm{Ha}][\mathrm{Mi}][\mathrm{ABS}]$ built up by tensoring supersingletons, ultrashort UIR's that describe the supermultiplet of five-brane coordinates transverse to the 5-brane world volume. [GiT] $[\mathrm{FeS} 1,2]$

However, the fact that $(2,0)$ conformal field theory is not a theory of point particles but is believed instead to be a theory of "tensionless strings" [W2][SW] should be reflected in the spectrum of "observable" conformal fields, possibly not the same as those that are detected in supergravity in $A d S_{7}$.

It is the aim of the present paper to emphasize a novel feature of conformal field theories in $d>4$, namely the fact that the spectrum of "short" primary conformal fields; that is, the limiting Harish Chandra modules that become reducible, is wider than what is naively obtained by "squaring" singleton representations [FlFr1] (massless conformal fields) [FFZ]. Tensionless $p$-branes bring up the subject of combining infinitely many massless fields with all spins. [Fr7][FV][Gu1][Sz]

A possible interpretation of such new fields is that they are "currents" related to "extended objects"; that is, that their space integrals measures the flux of an extended object in the boundary conformal field theory.

In the holographic picture such conformal current fields (of higher rank) should correspond to a new kind of bulk gauge fields in $A d S_{d+1}$. Antisymmetric (self-dual 
when $\frac{d}{2}$ is odd) singleton representations in any even dimension were found by the present authors [FeFr3] together with a new class of Harish-Chandra limiting unitary modules for $d-k$ forms of dimension $E_{0}=d-k\left(1 \leq k<\frac{d}{2}\right)$. All these modules are "zero center" modules, meaning that all the Casimirs vanish [FlFr3].

All other singletons (which are not zero center modules) were found by Siegel [Si] and also by Minwalla [Mi2] and Angelopoulos and Laoues [AL] by studying general "massless" conformal fields in $d$ dimensions. The latter authors also investigated their relations with Poincaré and de Sitter groups. These limiting Harish Chandra modules correspond to thresholds of the unitarity bound; the lowest values of $E_{0}$.

The general problem of classifying all highest weight modules of the simple Lie algebras was completed by Enright, Howe and Wallach [EHW]. Many special cases were known previously. Here we shall adapt the results of that paper to the physically interesting case of the conformal algebras $s o(2, d)$. We also discuss the algebras $s o^{*}(2 n)$. Some of the symplectic algebras have been studied already [Fr5][Gu1,2], while the unitary and the exceptional Lie algebras do not seem, at this time, to have found applications in physics. In the physics literature, the unitarity bounds of the $s o(2, d)$ algebra, corresponding to the limiting Harish Chandra modules classified in [EHW] were discussed much later: In relation to conformal field theories they were considered in Ref. [Mi2]. The same algebra in connection with gauge fields in $A d S_{2 n+1}$, and their behavior in the Poincaré (flat space) limit, was investigated in [DH] and more recently in [BMV]. References to other special results will be given.

There is no generally accepted definition of "masslessness" in higher dimension $[\mathrm{AFFS}][\mathrm{FeFr} 2][\mathrm{FFG}][\mathrm{L}][\mathrm{Me} 2]$. We propose that the most important property to be used for classification of field theories is whether or not they are gauge theories. A universal definition of "gauge theory" that we think is very natural is this: A field theory, invariant under a group or a supergroup $G$, is a gauge theory if the field or supermultiplet transforms by a non-decomposable representation of $G$. Such representations contain a maximal ideal of states with zero norm that constitutes the subspace of gauge modes. In 4 dimensions this property is strongly correlated with masslessness. (Exceptions: the massless scalar field is not a gauge theory and singletons are not massless.) The link between masslessness and gauge theories is strong in all dimensions whenever the group 
$G$ is the conformal group of the manifold.

It will be seen that there are two quite distinct types of ideals in the limiting Harish Chandra modules. Those that appear as cases II and III in the enumeration of $[\mathrm{EHW}]$ are of the singleton type. In odd dimensions ( $d$ odd) these are precisely the two singletons. In the bosonic case, both the Harish Chandra module and its ideal have highest weights that are trivial on the semisimple part of the compact subalgebra. In the $A d S_{d+1}$ field theory the full module is carried by the solutions of a Klein Gordon equation and the physical quotient is distinguished only by the boundary values. The situation in the fermionic case is similar. Those listed as types I,p include ordinary gauge theories of the vector/tensor type (Yang-Mills and gravity), in which the ideal appears as exact tensor fields (gradients). However, most of these are of mixed type. The ideal is not irreducible and its full characterization requires some specification of boundary conditions. The extent of complication that can arise may be appreciated by examining the case of the vector singleton in $A d S_{5}$, as was done in [FeFr1].

\section{2. $S O(2,2 n)$. Basis, highest weight modules.}

2.1. Basis. In this section and in the next one $G_{n}$, for $n=2,3, \ldots$, is the universal cover of the group $S O\left(\delta_{n}, \mathbf{R}\right)$, where $\delta_{n}$ is a symmetric, nonsingular 2-form with index $(2,2 \mathrm{n})$, and $\mathfrak{g}_{n}$ is the associated complexified Lie algebra. The compact subalgebra $\mathfrak{k}_{n}$ is isomorphic to the direct sum of $s o(2 n, \mathbf{R})$ and the real, one-dimensional Lie algebra.

Fix an index set $I=\left\{0,0^{\prime}, 1, \ldots, 2 n\right\}, \delta_{n}=\operatorname{Diag}\{-1,-1,1, \ldots, 1\}$, and a basis for $\mathfrak{g}_{n}$,

$$
\begin{gathered}
\left\{L_{a b}=-L_{b a} ; a, b \in I, a<b\right\}, \\
\left(L_{a b}\right)_{c}^{d}=\delta_{a}^{d} \delta_{b c}-a, b, \quad \delta_{b c}:=\left(\delta_{n}\right)_{b c}, a, b, c, d \in I .
\end{gathered}
$$

The commutation relations are $\left[L_{a b}, L_{c d}\right]=\delta_{b c} L_{d a}-a, b-c, d$. The compact subalgebra is generated by $\left\{L_{i j}, i, j=1, \ldots, 2 n\right\}$ and $L_{00^{\prime}}$.

We factor the space $M_{2+2 n}$ of $2+2 n$ dimensional matrices into a direct product $M_{2} \otimes M_{1+n}$. We introduce the Pauli matrices $\sigma_{1}, \sigma_{2}, \sigma_{3}$ in $M_{2}$, and the matrices

$$
\left(e_{i j}\right)_{k}^{l}=\delta_{i}^{l} \delta_{j k}, i, j, k, l \in\{0,1, \ldots, n\}
$$

in $M_{1+n}$. 
A Cartan subalgebra $\mathfrak{h}_{n}$ is generated by the set

$$
h_{i}=\sigma_{2} \otimes e_{i i}, i=0,1, \ldots, n .
$$

Positive/negative Serre generators,

$$
\begin{aligned}
& e_{ \pm i}=\frac{1 \pm \sigma_{2}}{2} e_{i, i+1}-\delta_{i i} \frac{1 \mp \sigma_{2}}{2} \otimes e_{i+1, i}, i=0,1, \ldots, n-1, \\
& e_{ \pm n}=\sigma_{1} \frac{1 \mp \sigma_{2}}{2} \otimes e_{n-1, n}-\frac{1 \pm \sigma_{2}}{2} \sigma_{1} \otimes e_{n, n-1}
\end{aligned}
$$

are associated with simple roots $\vec{r}(j), j=0,1, \ldots, n$, linear functions on $\mathfrak{h}_{n}$ defined by $\left[h_{i}, e_{j}\right]=r_{i}(j) e_{j}$. We find, for $i=0,1, \ldots, n$,

$$
\begin{aligned}
& {\left[h_{i}, e_{j}\right]=\left(\delta_{i j}-\delta_{i, j+1}\right) e_{j}, j=0,1, \ldots, n-1,} \\
& {\left[h_{i}, e_{n}\right]=\left(\delta_{i n}+\delta_{i, n-1}\right) e_{n} .}
\end{aligned}
$$

The positive roots are $r_{i}(j, k)=\delta_{i j} \pm \delta_{i k}, 0 \leq j<k \leq n$, and the half-sum of positive roots is

$$
\rho=\frac{1}{2} \sum \vec{r}(j, k)=(n, n-1, \ldots, 1,0) .
$$

Finally, we record the relations

$$
\begin{aligned}
& {\left[e_{i}, e_{-j}\right]=\delta_{i j}\left(h_{i+1}-h_{i}\right), i, j=0,1, \ldots, n-1,} \\
& {\left[e_{n}, e_{-j}\right]=-\delta_{n j}\left(h_{n}+h_{n-1}\right) .}
\end{aligned}
$$

2.2. Weights. A 'compact weight' will mean a dominant, integral weight on the Cartan subalgebra $\mathfrak{h}_{n}^{o}$ of $s o(2 n)$ generated by the set $h_{1}, \ldots, h_{n}$, namely

$$
w_{i}=w\left(h_{i}\right)=w_{i}, i=1, \ldots, n,
$$

where $w_{1}, \ldots, w_{n}$ are integers or half-integers satisfying

$$
w_{1} \geq w_{2} \geq \ldots \geq w_{n-1} \geq\left|w_{n}\right| .
$$

Each compact weight $\vec{w}$ is the highest weight of a finite dimensional, irreducible representation $D(\vec{w})$ of $s o(2 n)$. A 'weight' is a pair $(E, \vec{w})$ where $E \in \mathbf{R}$ is an eigenvalue of $\mathfrak{h}_{0}$ and $\vec{w}$ is a compact weight. 
2.3. Highest weight modules. A $\mathfrak{g}, \mathfrak{k}$ module is a representation of $\mathfrak{g}_{n}$ on a collection of finite dimensional $\mathfrak{k}_{n}$ modules. A Harish Chandra module is a $\mathfrak{g}, \mathfrak{k}$ module that is generated from a highest weight $\left(E_{0}, \vec{w}\right)$. Since $E$ will have the interpretation of energy, normally bounded below, "highest" will mean that $E_{0}$ is the lowest value of $E$. More precisely, consider the decomposition

$$
\mathfrak{g}_{n}=\mathfrak{g}_{-}+\mathfrak{k}_{n}+\mathfrak{g}_{+},
$$

defined by the grading of $\mathfrak{g}_{n}$ by the adjoint action of $h_{0}$. Thus elements of $\mathfrak{g}_{-}\left(\mathfrak{g}_{+}\right)$lower (raise) the energy. Fix $(E, \vec{w})$ and let $V_{0}$ be the associated $\mathfrak{k}_{n}$ module, promoted to a $\mathfrak{k}_{n}+\mathfrak{g}_{-}$module by letting $\mathfrak{g}_{-}$act trivially. Then the Harish Chandra module $V(E, \vec{w})$ is the space $U\left(\mathfrak{g}_{n}\right) \otimes^{\prime} V_{0}$, with the natural left action of $\mathfrak{g}_{n}$. The prime on $\otimes^{\prime}$ means that $\forall x \in \mathfrak{k}_{n}+\mathfrak{g}_{-}, x \otimes^{\prime}=\otimes^{\prime} x$.

Fix a compact weight $\vec{w}$. Consider the family of Harish Chandra modules $V\left(E_{0}, \vec{w}\right)$ with highest weight $\left(E_{0}, \vec{w}\right), E_{0} \in \mathbf{R}$. For $E_{0}$ large enough this representation is irreducible and unitarizable. The problem is to determine the values of $E_{0}$ such that

(a) The Harish Chandra module is reducible, with a maximal ideal $I\left(E_{0}, \vec{w}\right)$, and

(b) The quotient $D\left(E_{0}, \vec{w}\right)=V\left(E_{0}, \vec{w}\right) / I\left(E_{0}, \vec{w}\right)$ is unitarizable.

2.4. Results. Complete results for the case $n=2$ were obtained long ago by physicists. [Mac] The general solution is in [EHW]. We need to distinguish a number of different cases.

Let $\Delta_{c}(\vec{w})$ be the set of positive roots $\vec{r}$ such that $\langle\vec{w} \mid \vec{r}\rangle:=\sum_{i} w_{i} r_{i}=0$. It turns out to be one of the following:

Case I,p: $\Delta_{c}(\vec{w})$ is the root system of $s u(p), p=1, \ldots, n$. (When $p=1$, then this is the empty set.) Then $\Delta_{c}(\vec{w})$ contains the roots generated by the simple roots $\vec{r}(1), \ldots, \vec{r}(p-1)$, and this case is characterized by $\langle\vec{w}, \vec{r}(i)\rangle=0, i=1, \ldots, p-1$ and $\langle\vec{w}, \vec{r}(p)\rangle \neq 0$, or $w_{1}=w_{2}=\ldots=w_{p}>w_{p+1}$. (Here and below, when $p=n$, replace the last inequality by $>0$; when $p=n-1$, by $\left|w_{n}\right|$.)

Case II: $\Delta_{c}(\vec{w})$ is the root system of $s o(2 n)$. Then $\vec{w}=0$. 
The result is that the following is an exhaustive list of highest weights $\left(E_{0}, \vec{w}\right)$ that satisfy conditions (a),(b) above.

Case I,p: $w_{1}=w_{2}=\ldots=w_{p}>w_{p+1}, E_{0}=2 n-1+w_{1}-p ; p=1, \ldots, n$. The lowest energy of the maximal ideal is $E_{0}+1$.

Case IIa, IIb: $\vec{w}=0, E_{0}=n-1$ or 0 . The lowest energy of the maximal ideal is $E_{0}+2\left(\right.$ when $\left.E_{0}=n-1\right)$ or 1 (in the other case).

In each case, except the case when $E_{0}=0$, this value of $E_{0}$ marks the lower bound for unitary representations, and there are no other unitary, irreducible representations. The special case $D(0, \overrightarrow{0})$ is the identity representation; it is isolated in the family of highest weight, unitarizable representations.

\section{3. $S 0(2,2 n)$ invariant gauge theories.}

3.1. Field modules. Let $M$ be a $G_{n}$ homogeneous space, $\mathcal{P}$ a vector bundle over $M$ with finite dimensional fiber $F$ with a structure of $G_{n}$ module, and $\psi$ a covariant field on $M$, valued in $V$. We mean by this that we are considering a space $V$ of sections of $\mathcal{P}$ that admits an action of $G_{n}$ induced by the actions on $M$ and $F$. Without specifying this space in detail, we assume that it is a $\mathfrak{g}, \mathfrak{k}$-module, and that there is a subspace that has the properties of the space $V_{0}$ in subsection 2.3. Thus $V_{0}$ is finite dimensional, carries an irreducible representation of $\mathfrak{k}_{n}$ with highest weight $\left(E_{0}, \vec{w}\right)$, and is annihilated by $\mathfrak{g}_{-}$. Note that here $\mathfrak{k}_{n}$ may act only on $M$, only on $F$, or on both. The problem is to determine whether the action of $\mathfrak{g}_{n}$ on $V_{0}$ generates a decomposable $\mathfrak{g}_{n}$ module with a unitary quotient. For this to be the case it is necessary, but not sufficient, that the highest weight be one of the types listed in subsection 2.4.*

To proceed it is necessary to choose the manifold $M$. The one that is most likely to be of interest is the hyperboloid

$$
\sum \delta_{a b} y^{a} y^{b}=1
$$

* This has been our strategy for localizing "massless" fields in $A d S_{4}$. See for example $[\mathrm{Fr} 1][\mathrm{FeFr} 1]$. 
in the pseudo-Euclidean space $\mathcal{E}_{n}$ of dimension $2+2 n$ with metric $\delta_{n}$. Instead of functions on the hyperboloid, it is more convenient to consider homogenous functions on $\mathcal{E}_{n}$. The vector fields that implement the action of $\mathfrak{g}_{n}$ are

$$
-L_{a b}=y_{a} \partial_{b}-a, b, \quad y_{a}:=\delta_{a b} y^{b}, a, b \in I .
$$

It is helpful to introduce a time coordinate $t$, by the polar decomposition

$$
y_{ \pm}:=y^{o} \pm i y^{o^{\prime}}=: Y e^{ \pm i t} .
$$

Then

$$
h_{0}=i \frac{\partial}{\partial t} .
$$

Eigenfunctions of $h_{0}$ have the form $\left(y_{+}\right)^{\alpha}\left(y_{-}\right)^{\beta} \psi\left(y_{1}, \ldots, y_{2 n}\right)$ with eigenvalue $E=\beta-\alpha$. The subalgebras $\mathfrak{g}_{ \pm}$(energy raising and lowering operators) are spanned by

$$
\begin{aligned}
& E_{j}=L_{0 j}-i L_{0^{\prime} j}=2 y_{j} \partial_{+}+y_{-} \partial_{j}+\tilde{E}_{j}, \\
& E_{-j}=L_{0 j}+i L_{0^{\prime} j}=y_{+} \partial_{j}+2 y_{j} \partial_{-}+\tilde{E}_{-j},
\end{aligned}
$$

where $\tilde{E}_{ \pm j}$ are the matrices that represent the action in $F$. Choosing the degree of homogeneity equal to $-E_{0}$, we obtain a simple representation for the functions that belong to the ground states. For example, if $F$ is 1-dimensional it is

$$
\left(y_{+}\right)^{-E_{0}} .
$$

Now we can investigate the Harish Chandra module. From [EHW] we learn that the highest weight of the ideal is of the form $\left(E_{0}+1, \vec{w}^{\prime}\right)$ in case I and in case II when $E_{0}=0$, and of the form $\left(E_{0}+2, \vec{w}^{\prime}\right)$ in case II when $E_{0}=n-1$.

3.2. Case I. The highest weight of the ideal lies in the space obtained from the ground states by applying one raising operator. At this level of energy $\mathfrak{k}_{n}$ acts by the representation $D_{2 n} \otimes D(\vec{w})$, where $D_{2 n}$ is the defining representation of $s o(2 n)$. Let $\left\{v_{r}\right\}$ be a basis for the subspace $V_{0}$ associated with the highest weight, orthonomal with respect to the invariant inner product and making the matrices of $\mathfrak{k}_{n}$ diagonal. Then $\left\{E_{i} v_{r}\right\}$ is 
a basis for the subspace with energy $E_{0}+1$. In this subspace there are vectors of zero invariant norm if and only if the determinant of the matrix

$$
M_{i r}^{j s}=\left\langle v_{s}, E_{-j} E_{i} v_{r}\right\rangle \propto \delta_{r}^{s}\left(\left[E_{i}, E_{-j}\right]\right)_{r r} .
$$

vanishes. (Since $\left[E_{i}, E_{-i}\right]$ lies in the compact Cartan subalgebra, typically $\left[E_{i}, E_{-i}\right]=$ $h_{i+1}-h_{i}$, one easily understands why repetitions among the components of the compact weights are characteristic of the highest weights of reducible Harish Chandra modules.)

3.3. Case IIa, $E_{0}=n-1$. The highest weight is $(n-1, \overrightarrow{0})$, and the associated function is

$$
f(y)=\left(y_{+}\right)^{1-n}
$$

We apply two raising operators to get

$$
f_{j k} \propto\left(y_{-} \partial_{j}+2 y_{j} \partial_{+}\right) y_{k}\left(y_{+}\right)^{-n}=\delta_{j k} y_{-}\left(y_{+}\right)^{-n}-2 n y_{j} y_{k}\left(y_{+}\right)^{-n-1} .
$$

The problem is to determine the structure of this space as a $\mathfrak{k}_{n}$ module. It is a sum of two irreducible representations, one of them one dimensional and spanned by the trace

$$
\sum_{j} f_{j j} \propto\left(\delta_{a b} y^{a} y^{b}\right)\left(y_{+}\right)^{-1-n}
$$

The first factor is the invariant that is constant on the hyperboloid and this function is the highest weight vector of $D(n+1, \overrightarrow{0})$. The subspace of functions generated from this one is an ideal and is a space of gauge modes, of zero invariant norm. The Harish Chandra module has the structure

$$
D(n-1, \overrightarrow{0}) \rightarrow D(n+1, \overrightarrow{0})
$$

The best known example is: $n=2$, the module is a space of solutions of the covariant Klein Gordon equation in $A d S_{5}$ [FeFr1]. If, instead of the hyperboloid, one passes to the cone (Dirac's cone, conformally compactified Minkowski space), then the functions in the ideal vanish and the representation becomes irreducible. This construction generalizes directly to $\mathrm{AdS}_{2 n+1}[\mathrm{FeFr} 1]$. 
3.4. Case IIb, $E_{0}=0$. Here $D\left(E_{0}, \vec{w}\right)=D(0, \overrightarrow{0})$ is the trivial representation. The maximal ideal in the Harish Chandra module has highest weight $(1, \vec{\alpha})$, where $\vec{\alpha}$ is the highest weight of the defining representation $D_{2 n}$ of $s o(2 n)$ (the vector representation). There are essentially two different ways that this representation can appear in a field theory. (a) The basis for the space of functions associated with the highest weight is the function 1; the trivial representation of $\mathfrak{g}_{n}$ appears as a direct summand. (b) The basis for the space of functions associated with the highest weight is the function $t \propto \log y_{+}$; the trivial representation is a proper quotient of a nondecomposable representation. Both cases are familiar from the analysis of conformal QED, $n=2$ [BFH1].

\section{4. $S O(2,2 n+1)$. Basis, highest weight modules.}

4.1. Basis. In this section and in the next one $G_{n}$, for $n=1,2, \ldots$, is the universal cover of the group $S O\left(\delta_{n}, \mathbf{R}\right)$, where $\delta_{n}$ is a symmetric, nonsingular 2-form with index $(2,2 \mathrm{n}+1)$, and $\mathfrak{g}_{n}$ is the associated complexified Lie algebra. The compact subalgebra $\mathfrak{k}_{n}$ is isomorphic to the direct sum of $s o(2 n+1, \mathbf{R})$ and the real, one-dimensional Lie algebra.

Fix an index set $I=\left\{0,0^{\prime}, 1, \ldots, 2 n, 2 n+1\right\}, \delta_{n}=\operatorname{Diag}\{-1,-1,1, \ldots, 1,1\}$, and a basis for $\mathfrak{g}_{n}$,

$$
\begin{gathered}
\left\{L_{a b}=-L_{b a} ; a, b \in I, a<b\right\}, \\
\left(L_{a b}\right)_{c}^{d}=\delta_{a}^{d} \delta_{b c}-a, b, \quad \delta_{b c}:=\left(\delta_{n}\right)_{b c}, a, b, c, d \in I .
\end{gathered}
$$

The commutation relations are $\left[L_{a b}, L_{c d}\right]=\delta_{b c} L_{d a}-a, b-c, d$. The compact subalgebra is generated by $\left\{L_{i j}, i, j=1, \ldots, 2 n+1\right\}$ and $L_{00^{\prime}}$. These are square matrices of dimension $2+2 n+1$. Let $M_{2+2 n+2}$ be the space of matrices obtained by adding a last row and a last column. We factor this space into a direct product $M_{2} \otimes M_{1+n}$. We introduce the Pauli matrices $\sigma_{1}, \sigma_{2}, \sigma_{3}$ in $M_{2}$, and the matrices

$$
\left(e_{i j}\right)_{k}^{l}=\delta_{i}^{l} \delta_{j k}, \quad i, j, k, l \in\{0,1, \ldots, n, n+1\}
$$

in $M_{1+n+1}$. Finally, we remove the last row and the last column. The last row of the matrices of dimension $2+2 n+1$ is now represented as the collection $u \otimes e_{n+1, i}$ and the last column as $v \otimes e_{i, n+1}$, where $u, v$ are rows, columns of length 2 , and $i$ runs over $\{0,1, \ldots, n\}$. 
A Cartan subalgebra $\mathfrak{h}_{n}$ is generated by the set

$$
h_{i}=\sigma_{2} \otimes e_{i i}, i=0,1, \ldots, n
$$

Positive/negative Serre generators are

$$
\begin{aligned}
& e_{ \pm i}=\frac{1 \pm \sigma_{2}}{2} e_{i, i+1}-\delta_{i i} \frac{1 \mp \sigma_{2}}{2} \otimes e_{i+1, i}, i=0,1, \ldots, n-1, \\
& e_{ \pm n}=\left(\begin{array}{c}
1 \\
\pm i
\end{array}\right) \otimes e_{n, n+1}-(1, \pm i) \otimes e_{n+1, n},
\end{aligned}
$$

are associated with simple roots $\vec{r}(j), j=0,1, \ldots, n$, linear functions on $\mathfrak{h}_{n}$ defined by $\left[h_{i}, e_{j}\right]=r_{i}(j) e_{j}$. We find, for $i=0,1, \ldots, n$,

$$
\begin{aligned}
& {\left[h_{i}, e_{j}\right]=\left(\delta_{i j}-\delta_{i, j+1}\right) e_{j}, j=0,1, \ldots, n-1,} \\
& {\left[h_{i}, e_{n}\right]=\delta_{i n} e_{n} .}
\end{aligned}
$$

The positive roots are $r_{i}(j, k)=\delta_{i j} \pm \delta_{i k}, 0 \leq j<k \leq n-1$, and $r_{i}(j)=\delta_{i j}$, and the half-sum of positive roots is

$$
\rho=\frac{1}{2} \sum \vec{r}(j, k)=\left(n+\frac{1}{2}, n-\frac{1}{2}, \ldots, \frac{1}{2}\right) .
$$

Finally, we record the relations

$$
\begin{aligned}
& {\left[e_{i}, e_{-j}\right]=\delta_{i j}\left(h_{i}-h_{i+1}\right), i=0,1, \ldots, n-1,} \\
& {\left[e_{n}, e_{-j}\right]=-2 \delta_{n j} h_{n} .}
\end{aligned}
$$

4.2. Weights. A 'compact weight' will mean a dominant, integral weight on the Cartan subalgebra $\mathfrak{h}_{n}^{o}$ of $s o(2 n+1)$ generated by the set $h_{1}, \ldots, h_{n}$, namely

$$
w_{i}=w\left(h_{i}\right)=w_{i}, i=1, \ldots, n,
$$

where $w_{1}, \ldots, w_{n}$ are integers or half-integers satisfying

$$
w_{1} \geq w_{2} \geq \ldots \geq w_{n} \geq 0 .
$$

Each compact weight $\vec{w}$ is the highest weight of a finite dimensional, irreducible representation $D(\vec{w})$ of $s o(2 n+1)$. A 'weight' is a pair $(E, \vec{w})$ where $E \in \mathbf{R}$ and $\vec{w}$ is a compact weight. 


\subsection{Highest weight modules. See subsection 2.3 .}

4.4. Results. The solution is given in [EHW]. The special case $n=1$ was done long ago by Evans [E]. We need to distinguish a number of different cases.

Let $\Delta_{c}(\vec{w})$ be the set of positive roots $\vec{r}$ such that $\langle\vec{w} \mid \vec{r}\rangle:=\sum_{i} w_{i} r_{i}=0$. It turns out to be one of the following:

Case I, $p(1 \leq p \leq n): \Delta_{c}(\vec{w})$ is the root system of $s u(p), p=1, \ldots, n$. (When $p=1$, then this is the empty set.) Then $\Delta_{c}(\vec{w})$ contains the roots generated by the simple $\operatorname{roots} \vec{r}(1), \ldots, \vec{r}(p-1)$, and this case is characterized by $\langle\vec{w}, \vec{r}(i)\rangle=0, i=1, \ldots, p-1$ and $\langle\vec{w}, \vec{r}(p+1)\rangle \neq 1$, or $w_{1}=w_{2}=\ldots=w_{p}>w_{p+1}$.

Case II: $\Delta_{c}(\vec{w})$ is the root system of $s o(2 n+1)$. Then $\vec{w}=0$.

Case III: $\Delta_{c}(\vec{w})$ is the root system of $s u(n), w_{1}=\ldots=w_{n}$.

The result is that the following is an exhaustive list of highest weights $\left(E_{0}, \vec{w}\right)$ that satisfy conditions (a),(b) above.

Case I,p: $w_{1}=w_{2}=\ldots=w_{p}>w_{p+1}, E_{0}=2 n+w_{1}-p ; p=1, \ldots, n$. The lowest energy of the maximal ideal is $E_{0}+1$.

Case IIa, IIb: $\vec{w}=0, E_{0}=n-\frac{1}{2}$ or 0 . The lowest energy of the maximal ideal is $E_{0}+2$ or 1 , respectively.

Case III: $w_{1}=\ldots=w_{n}=\frac{1}{2}, E_{0}=n$. The lowest energy of the maximal ideal is $E_{0}+1$.

In each case, except when $E_{0}=0$, this value of $E_{0}$ marks the lower bound for unitary representations, and there are no other unitary, irreducible representations.

\section{5. $S 0(2,2 n+1)$ invariant gauge theories.}

5.1. Field modules. See subsection 3.1. The problem is to determine whether the action of $\mathfrak{g}_{n}$ on $V_{0}$ generates a decomposable $\mathfrak{g}_{n}$ module with a unitary quotient. For this to 
be the case it is necessary, but not sufficient, that the highest weight be one of the types listed in subsection 4.4.

To get further it is necessary to choose the manifold $M$. The one that is most likely to be of interest is the hyperboloid

$$
\sum \delta_{a b} y^{a} y^{b}=1
$$

in the pseudo-Euclidean space $\mathcal{E}_{n}$ of dimension $2 n+3$ with metric $\delta_{n}$. See subsection 3.1.

From [EHW] we learn that the highest weight of the ideal is of the form $\left(E_{0}+1, \vec{w}^{\prime}\right)$ in Case I and in Case III, and in Case II when $E_{0}=0$, and of the form $\left(E_{0}+2, \vec{w}^{\prime}\right)$ in Case II when $E_{0}=n-\frac{1}{2}$.

5.2. Case I. The highest weight of the ideal lies in the space obtained from the ground states by applying one raising operator. At this level of energy $\mathfrak{k}_{n}$ acts by the representation $D_{2 n+1} \otimes D(\vec{w})$, where $D_{2 n+1}$ is the defining representation of $s o(2 n+1)$. See subsection 3.2.

5.3. Case IIa, $E_{0}=n-\frac{1}{2}$. The highest weight is $\left(n-\frac{1}{2}, \overrightarrow{0}\right)$, and the associated function is

$$
f(y)=\left(y_{+}\right)^{\frac{1}{2}-n}
$$

We apply two raising operators to get

$$
f_{j k} \propto\left(y_{-} \partial_{j}+2 y_{j} \partial_{+}\right) y_{k}\left(y_{+}\right)^{-\frac{1}{2}-n}=\delta_{j k} y_{-}\left(y_{+}\right)^{-\frac{1}{2}-n}-(2 n+1) y_{j} y_{k}\left(y_{+}\right)^{-\frac{3}{2}-n} .
$$

The problem is to determine the structure of this space as a $\mathfrak{k}$ module. It is a sum of two irreducible representations, one of them one dimensional and spanned by the trace

$$
\sum_{j} f_{j j} \propto\left(\delta_{a b} y^{a} y^{b}\right)\left(y_{+}\right)^{-\frac{3}{2}-n}
$$

The first factor is the invariant that is constant on the hyperboloid and this function is the highest weight vector of $D\left(n+\frac{3}{2}, \overrightarrow{0}\right)$. The subspace of functions generated from this one is an ideal and is a space of gauge modes of zero invariant norm. The Harish Chandra module has the structure

$$
D\left(n-\frac{1}{2}, \overrightarrow{0}\right) \rightarrow D\left(n+\frac{3}{2}, \overrightarrow{0}\right)
$$


The best known example is: $n=1$, the module is a space of solutions of the covariant Klein Gordon dipole equation in $A d S_{4}$, the bosonic singleton [FlFr2], instead of the hyperboloid, one passes to the cone (Dirac's cone, conformally compactified Minkowski space), then the functions in the ideal vanish and the representation becomes irreducible. This constrution generalizes directly to $\mathrm{AdS}_{2 n+2}$.

Case $I I b, E_{0}=0$. Here $D\left(E_{0}, \vec{w}\right)=D(0, \overrightarrow{0})$ is the trivial representation. The maximal ideal in the Harish Chandra module has highest weight $(1, \vec{\alpha})$, where $\vec{\alpha}$ is the highest weight of the defining representation $D_{2 n+1}$ of $s o(2 n+1)$ (the vector representation). There are two different ways that this representation can appear in a field theory, precisely as described in the case of $s o(2,2 n)$. Both cases are familiar from the analysis of $A d S_{4} \mathrm{QED}, n=1 \quad[\mathrm{FH}]$.

Case III, $w_{1}=\ldots w_{n}=\frac{1}{2}, E_{0}=1$. This is other singleton representation. The case $n=1$ is the familiar fermionic singleton. In this case the two singletons combine to a representation of $O s p(1 / 4)$ [Fr3] and singleton multiplets combine to form a representation of $O \operatorname{sp}(N / 4) \quad[\mathrm{FN}][\mathrm{BSST}][\mathrm{BD}][\mathrm{NST}]$. For $n>1$, it was discovered by [AL] that there are just two singletons

\section{6. $S O^{*}(2 n)$. Basis, highest weight modules.}

6.1. Basis. In this section and in the next one $G_{n}$, for $n=2,3, \ldots$, is the universal cover of the group $S O^{*}(2 n)$ of $2 n$ dimensional, unimodular matrices that preserve the hermitean form $\sigma_{2} \otimes \mathbf{1}_{n}$, and $\mathfrak{g}_{n}$ is the associated complexified Lie algebra. The compact subalgebra $\mathfrak{k}_{n}$ is isomorphic to $u(n)$.

Fix an index set $I=\{1, \ldots, 2 n\}, \delta_{n}=\operatorname{Diag}\{1, \ldots, 1\}$, and a basis for $\mathfrak{g}_{n}$,

$$
\begin{gathered}
\left\{L_{a b}=-L_{b a} ; a, b \in I, a<b\right\} \\
\left(L_{a b}\right)_{c}^{d}=\delta_{a}^{d} \delta_{b c}-a, b, \quad \delta_{b c}:=\left(\delta_{n}\right)_{b c}, a, b, c, d \in I .
\end{gathered}
$$

The commutation relations are $\left[L_{a b}, L_{c d}\right]=\delta_{b c} L_{d a}-a, b-c, d$. The compact subalgebra is the unitary subalgebra. 
We factor the space $M_{2 n}$ of $2 n$ dimensional matrices into a direct product of $M_{2} \otimes$ $M_{n}$. We introduce the Pauli matrices $\sigma_{1}, \sigma_{2}, \sigma_{3}$ in $M_{2}$, and the matrices

$$
\left(e_{i j}\right)_{k}^{l}=\delta_{i}^{l} \delta_{j k}
$$

in $M_{n}$.

A Cartan subalgebra $\mathfrak{h}_{n}$ is generated by the set

$$
\begin{aligned}
& h_{0}=\frac{1}{2 n}\left(e_{11}+\ldots+e_{n n}\right), \\
& h_{i}=\sigma_{2} \otimes e_{i i}-2 \mathfrak{h}_{0}, i=1, \ldots, n .
\end{aligned}
$$

In this section and in the next one $\delta_{i j}$ is the Kroenecker symbol. Positive/negative Serre generators,

$$
\begin{aligned}
& e_{ \pm i}=\frac{1 \pm \sigma_{2}}{2} e_{i, i+1}-\frac{1 \mp \sigma_{2}}{2} \otimes e_{i+1, i}, i=1, \ldots, n-1, \\
& e_{ \pm n}=\sigma_{1} \frac{1 \mp \sigma_{2}}{2} \otimes e_{n-1, n}+\frac{1 \pm \sigma_{2}}{2} \sigma_{1} \otimes e_{n, n-1},
\end{aligned}
$$

(those in the first line compact) are associated with simple roots $\vec{r}(j), j=1, \ldots, n$, linear functions on $\mathfrak{h}_{n}$ defined by $\left[h_{i}, e_{j}\right]=r_{i}(j) e_{j}$. We find, for $i=1, \ldots, n$,

$$
\begin{aligned}
{\left[h_{i}, e_{j}\right] } & =\left(\delta_{i j}-\delta_{i, j+1}\right) e_{j}, j=1, \ldots, n-1, \\
{\left[h_{i}, e_{n}\right] } & =\left(\delta_{i n}+\delta_{i, n-1}\right) e_{n} .
\end{aligned}
$$

The positive roots are $r_{i}(j, k)=\delta_{i j}-\delta_{i k}, 1 \leq j<k \leq n$ (compact) $r_{i}(j, k)=\delta_{i j}+\delta_{i k}, 1 \leq$ $j<k \leq n$ (noncompact) and the half-sum of positive roots is

$$
\rho=\frac{1}{2} \sum \vec{r}(j, k)=(n-1, \ldots, 1,0) .
$$

6.2. Weights. A 'compact weight' will mean a dominant, integral weight on the Cartan subalgebra $s u(n)$ generated by the set $h_{1}, \ldots, h_{n}$, namely

$$
w_{i}=w\left(h_{i}\right)=w_{i}, i=1, \ldots, n, w_{1}+\ldots+w_{n}=0
$$

where $w_{1}, \ldots, w_{n}$ are integers or half integers and $w_{1} \geq \ldots \geq w_{n}$. Each compact weight $\vec{w}$ is the highest weight of a finite dimensional, irreducible representation $D(\vec{w})$ of $s u(n)$. A 'weight' is a pair $(E, \vec{w})$ where $E \in \mathbf{R}$ is an eigenvalue of $h_{0}$ and $\vec{w}$ is a compact weight. 


\subsection{See subsection 2.3 .}

6.4. Results. The solution was found by Enright, Howe and Wallach [EHW]. We need to distinguish several cases.

Case I,q, $w_{2}=w_{3}=\ldots=w_{q+1}, E_{0}=2 n-3+w_{2}-q ; q=1, \ldots, n-1$. The lowest energy of the maximal ideal is $E_{0}+1$.

Case II,p: $w_{1}=\ldots=w_{p}, p=3, \ldots, n$, with $E_{0}=2 n-3+w_{1}-p, p$ even, $E_{0}=2 n-2+w_{1}-p, p$ odd. The lowest energy of the maximal ideal is $E_{0}+\left[\frac{p}{2}\right]$.

In both of these cases, this value of $E_{0}$ marks the lower bound for unitary representations. But here there are additional, isolated, unitary representations ( for $n>$ ), namely

Case 3: Same as case II, except that $E_{0}$ takes one of the values

$$
E_{0}=2 n+w_{1}-2 p+2 j, \quad 0 \leq 2 j \leq\left\{\begin{array}{l}
p-4, p \text { even } \\
p-5, p \text { odd }
\end{array},\right.
$$

with $j$ integer. This happens is when $n=p=4, \vec{w}=0, E_{0}=0$. The first non trivial case is $n=5, p=4, E_{0}=w_{1}+2$.

\section{The six-dimensional case.}

In this section we apply the results to the case of $A d S_{7}$, with a 6 -dimensional Minkowski boundary, where the conformal algebra is $s o(2,6) \approx s o^{*}(8)$. To label the highest weight of the compact subalgebra $s o(6) \approx s u(4)$ we shall use the Dynkin labels $a_{1}, a_{2}, a_{3}$, related to the highest weight $\vec{w}$ in the following way:

$$
2 w_{1}=a_{1}+a_{3}+2 a_{2}, 2 w_{2}=a_{1}+a_{3}, 2 w_{3}=a_{3}-a_{1} .
$$

The highest weight of the Harish Chandra modules will be indicated by the quadruple $E_{0}, a_{1} \cdot a_{2}, a_{3}$. The three first classes of unitary representations listed in subsection 2.4 as Cases $I, p=1,2,3$ are:

$$
\begin{aligned}
& p=1: E_{0} \geq 4+w_{1}=4+a_{2}+\left(a_{1}+a_{3}\right) / 2, \quad a_{2} \neq 0, \\
& p=2: E_{0} \geq 3+w_{1}=3+\left(a_{1}+a_{3}\right) / 2, \quad a_{2}=0, \\
& p=3: E_{0} \geq 2+w_{1}=2+a_{1} / 2, \quad a_{2}=a_{3}=0, \text { or } 1,3 \rightarrow 3,1 .
\end{aligned}
$$


The irreducible representations at the bound of the third class are the singletons, found in $[\mathrm{Si}][\mathrm{Mi} 2][\mathrm{AL}]$ and associated with massless fields on the boundary. Later they were discussed, in the context of the $O \operatorname{sp}\left(8^{*} / 4\right)$ superalgebra, in the papers [GuT],[FeS1]. In a recent paper the extension of the unitary modules corresponding to $p=1$ and $p=3$, to the superalgebra $O \operatorname{sp}\left(8^{*} / 4\right)$, was found [FeS2].

Notice that the bound in the first case is twice the bound in the third case. The limiting Harish Chandra modules in Case $p=1$ can be constructed by squaring the singleton representations. This is the usual situation, where massless fields in the bulk correspond to (conserved) tensor currents on the boundary, that are bilinears in boundary massless fields [FeFr2]. These two series, $p=1,3$ (for $a_{3}=0$ ) were investigated in [FeFr3] and in [FeS1].

The series in the intermediary case, $p=2$, has no analogue in 4 dimensions. What is new here, in six dimensions, is that, for $p=2$, the unitary bound on $E_{0}$ is lower than the conformal degree of the conserved singleton currents. The fields associated with the unitary bound in case $p=1$ are neither elementary massless, nor composite. The singletons with $E_{0}=3, a_{1}=2(p=3)$, and the operators with $E_{0}=4, a_{1}=a_{3}=1$ $(p=2)$ were discussed in [FeFr3].

The unitary quotient of the Harish Chandra module $D\left(E_{0}, 1,0,1\right)$, with $E_{0}=4$, $a_{1}=a_{3}=1, a_{2}=0$ can be represented as a closed 4 -form, $d J_{4}=0$, or its conserved dual $J_{4}^{*}=J_{2}$,

$$
\left(d^{*} J_{2}\right)_{\mu}=\partial^{\nu} J_{\mu \nu}=0
$$

The integral

$$
\int_{\mathcal{M}_{4}} J_{4}=Q_{1}
$$

defines the flux of a string in six dimensions, so $J_{4}$ is the current operator that is related to "tensionless" strings $[\mathrm{W} 2][\mathrm{SW}][\mathrm{Sa}][\mathrm{DFKR}][\mathrm{DLP}]$.

This also explains why* we do not have a simple candidate for such an operator; it exists as a consequence of the tensionless string interaction, and it cannot be described

* Moreover, the string is self dual, which means that it is not only dyonic, but that its electric and magnetic charges are equal. In this respect there is a distinction between even and odd values of $n$, since for odd $n$ the singleton representation $p=n$ is real, while for even $n$ it is complex. 
as a local, bilinear in the massless (singleton) fields.

The picture presented here suggests that there could be a bulk theory that generalizes supergravity by including this new bulk field, with interactions.*

Such a current $J_{4}$ is actually known in 6-dimensional $(1,0)$ supersymmetric theories [Sa] where, away from the conformal point, tensor multiplets interact with non abelian gauge fields and it takes the form [Sa][FRS][DFKR]

$$
J_{4}=\operatorname{Tr} F \wedge F, \quad Q_{1}=\int \operatorname{Tr} F \wedge F .
$$

The string flux is related to the instanton number (instanton in a space transverse to the string). [DLP] The flat limit of a tensionless string was discussed in [DLLP].

\section{Speculations about higher dimensions.}

For higher dimensions, with $d-2$ a multiple of 4 , we may think that the above formulas generalize to $(d=2 n) J_{n+1}=\operatorname{Tr} F^{\frac{n+1}{2}}$, so that

$$
Q_{n-2}=\int_{\mathcal{M}_{n+1}} \operatorname{Tr} F^{\frac{n+1}{2}},
$$

which is the $\frac{n+1}{2}$ Chern class of the gauge group.

The unitary bound for the family $D\left(E_{0}, \vec{w}\right)$ of Harish Chandra so $(2,2 n)$ modules, for a fixed, integral, dominant compact weight $\vec{w}$ was obtained in subsection 2.4. We concentrate on the cases,

$$
w_{1}=\ldots w_{p}>\begin{gathered}
w_{p+1}, \quad p<n \\
0, \quad p=n
\end{gathered}
$$

where the bound is

$$
E_{0}=2 n-1+w_{1}-p
$$

Singletons are in the class $p=n$.

The particular case $w_{1}=1, E_{0}=d-p$, was investigated in [FeFr3]. The fields are closed $d-p$ forms,

$$
d J_{d-p}=0 .
$$

* However, the unitarity bounds of $O \operatorname{sp}\left(8^{*} / 2 N\right)$ superalgebras seem to exclude this possibility [Mi2][FeS2]. 
Indeed, this equation is conformally invariant [W1] if the conformal degree is $d-p$.

The integral

$$
Q_{p-1}=\int_{\mathcal{M}_{d-p}} J_{d-p}
$$

is the flux of a $p-1$ brane $(d-p$ are the coordinates transverse to the brane) that we interpret as tensionless $p$-1-brane, a natural generalization from the 6 -dimensional case.

Let us consider a hypothetical $d=10$ dimensional conformal field theory, the holographic description of a hypothetical 11 dimensional theory in $A d S_{11}$. [Gu1][Ho] The bosonic singleton, other than the scalar, is a self dual five form with $E_{0}=5$. However, there is a whole set of unitary Harish Chandra modules that are all zero center modules (all the Casimir operators of the conformal group vanish on them), described by a 4 -form with $E_{0}=6$, a 3 -form with $E_{0}=7$, a 2 -form with $E_{0}=8$ and a vector with $E_{0}=9 .(p=1,2,3,4$. $)$ The integrals of these currents may produce fluxes for 3,2,1 and 0 branes, respectively. The last is the usual global charge present in all theories (for any $d \geq 3$ ). Therefore, a 10 dimensional conformal field theory may be considered as a theory of tensionless 3,2 , and 1 branes [FP].

It is tempting to go on to suggest that the only conformal branes are the dyonic (self dual) 3-branes, analogues of the dyonic string in dimension 6. In support of this there is the obvious fact, already observed in [FeFr3], that this theory resembles a kind of conformal limiting case of IIB supergravity. In analogy with the 6 dimensional case we may think that such a theory, away from the conformal point, is defined thropugh non abelian gauge interactions, so that the 6 -form current is of the type

$$
J_{6}=\operatorname{Tr} F^{3}
$$

where $F$ is a non abelian 10 dimensional gauge field. In such a situation the 3-brane charge would be related to a topological configuration of the gauge field with non vanishing third Chern class

$$
Q_{3}=\int \operatorname{Tr} F^{3}
$$

It is not known whether the above considerations may be accommodated in a supersymmetric theory. To answer this question further studies on new types of supersymmetric structures in higher dimensions may be needed. 


\section{Appendix. Case by case.}

\section{A.1. $S O(2,2 n)$.}

$\underline{n=1}$. Since $S O(2,2)$ is not simple, this case is not encompassed by the investigation of [EHW]. The results apply nevertheless if we interpret as follows. Take a standard Chevalley basis for each of the two factors $S O(2,1)$, with generators $A, B$ of the compact Cartan generators normalized so that the weights are \pm 1 in the adjoint representation. Set $h_{0}=(A+B) / 2, h_{1}=(A-B) / 2$. Let $(a, b)$ be a generic pair of eigenvalues of $(A, B)$, then our highest weight is given by $E_{0}=(a+2) / 2, w_{1}=(a-b) / 2$.

Case I, 1 is the case $b=1, E_{0}=w_{1}>0$. The Harish Chandra module is irreducible on the first $S O(2,1)$ factor and equivalent to $D(0) \rightarrow D(1)$ on the other. This representation appears in the gauge theory of singletons in $2+1$ dimensions [Fr6][FlFr4].

Case II is $a=b=0, E_{0}=w_{1}=0$. The quotient is $D(0,0)$ and the ideal is $D(1,1), D(1,-1)$ or both.

$\underline{n=2 .} S O(2,4)$ is the anti De Sitter group in 5 dimensions and the conformal group in 4 dimensions. The compact subgroup is $S O(4)=S U(2) \otimes S U(2)$. The usual notation for the compact weights is $\left(J_{1}, J_{2}\right)$ and is adapted to this decomposition, with $J_{1}, J_{2}$ integral or half integral. Set $w_{1}=J_{1}+J_{2}, w_{2}=J_{1}-J_{2}$.

Case I, 1 is the general case, $w_{1}>w_{2}$ or $J_{1} J_{2} \neq 0$. The formula $E_{0}=2 n-1+w_{1}-p$ becomes $E_{0}=J_{1}+J_{2}+2$. The lowest energy of the ideal is $E_{0}+1$. The simplest field theory in $A d S_{5}$, with $J_{1}=J_{2}=\frac{1}{2}$, is the theory of a vector field, homogeneous of degree -3 . The ground states are

$$
f_{i}(y)=\left(y_{+}\right)^{-3} \epsilon_{i}, \quad i=1,2,3,4,
$$

with $\epsilon_{i}$ constant. The lowest weight of the ideal is $(4,0,0)$ and the associated gauge field subspace consists of the gradients or exact vector fields. The next simplest cases are the fields of $A d S_{5}$ supergravity, with highest weights $\left(E_{0}, w_{1}, w_{2}\right)$ equal to $(4,1,1)$ and $\left(\frac{7}{2}, \frac{1}{2}, 1\right),\left(\frac{7}{2}, 1, \frac{1}{2}\right)$. 
Case I,2 is characterized by $w_{1}=w_{2}$ or $J_{2}=0$. The formula for $E_{0}$ becomes $E_{0}=J_{1}+1$. This is the familiar case of conformally invariant field theories in 4dimensional Minkoski space (or $A d S_{4}$ ) [BFH1]. Realized as field theories in $A d S_{5}$ they are topological singleton field theories [FeFr1].

Case IIa has $w_{1}=w_{2}=0$ and $E_{0}=1$. It is the representation associated with a scalar, massless field in 4 dimensions. Case IIb is the case $E_{0}=J_{1}=J_{2}=0$. The ideal has highest weight $(1,1,0)$; it is the highest weight of a non unitary irreducible representation.

$\underline{n=3 .} S O(2,6)$ is the symmetry group of $A d S_{7}$. See Section 7 .

\section{A.2. $S O(2,2 n+1)$.}

$\underline{n=1}$. The nondecomposable representations of $S 0(2,3)$, and the associated field theories in $A d S_{4}$ have been studied extensively.

Case I,1: $w_{1}>0, E_{0}=w_{1}+1$. The highest weight of the ideal is $w_{1}+2, w_{1}-1$. These are the representations associated with massless fields with spin greater than or equal to $1[\mathrm{BFH} 2,3][[\mathrm{FFr} 1][\mathrm{Fr} 2][\mathrm{FH}]$.

Case IIa: $w_{1}=0, E_{0}=\frac{1}{2}$. The highest weight of the ideal is $\left(\frac{5}{2}, 0\right)$. This is the bosonic singleton, described by a scalar field [FIFr2].

Case IIb: The Harish Chandra module is $D(0,0) \rightarrow D(1,1)$; the ideal non unitary. This is a component of the field representation of QED in $A d S_{4}$. [BFH2]

Case III: $w_{1}=\frac{1}{2}, E_{0}=1$; the fermionic singleton, described by a spinor field $[\mathrm{He}][\mathrm{P}]$.

$\underline{n=2 .} S O(2,5)$ is the symmetry group of $A d S_{6}$.

Case I,1: $w_{1}>w_{2}, E_{0}=3+w_{1}$.

Case I,2: $w_{1}=w_{2}>0, E_{0}=2+w_{1}$.

Case IIa,IIb: $\vec{w}=0, E_{0}=\frac{3}{2}$, the bosonic singleton, or 0 . 
Case III: $w_{1}=w_{2}=\frac{1}{2}, E_{0}=2$, the fermionic singleton. This case is particularly interesting since it is related to the exceptional F(4) superconformal algebra $[\mathrm{N}][\mathrm{R}]$. The spin zero and spin one-half singletons are combined in the singleton hypermultiplet.

\section{A.3. $S O^{*}(2 n)$.}

The isomorphisms $s o^{*}(4)=s o(2,1) \times s o(3), s o^{*}(6)=s u(1,3)$ and $s o^{*}(8)=s o(2,6)$ allow us to omit the cases $n=2,3,4$. We do not discuss the $n>4$ cases.

\section{Acknowledgements.}

The work of S.F. has been supported in part by the European Commision TMR programme ERBFMRX-CT96-0045 (Laboratori Nazionali di Frascati, INFN) and by DOE grant DE-FG03-91ER40662, Task C.

\section{References.}

[ABS] Aharony, O., Berkooz, N. and Seiberg, N., "Lightcone description of $(2,0)$ superconformal theories in six dimensions", Adv. Theor. Math. Phys. 2 (1998) 119. (hep-th/9712117)

[A0Y] Aharony, O., Oz, Y. and Yin, Z., "M-theory on $A d S(p) \times S(11-p)$ and superconformal field theories", Phys. Lett. B430 (1998) 87. (hep-th/9803051)

[AL] Angelopoulos, E. and Laoues, M., "Massless in $n$ dimensions", Rev. Math. Phys. 10 (1998) 271. (hep-th/9806199)

[AFFS] Angelopoulos, E., Flato, M., Fronsdal, C. and Sternheimer, D., " Massless particles, conformal group and De Sitter Universe", Phys. Rev., D23 (1981) 1278.

[BSST] Bergshoeff, E.D., Salam, A. Sezgin, E. and Tanii, Y. "Singletons, higher spin massless states and the super membrane", Phys. LettB205 (1998) 137.

[BD] Blencowe, M.P and Duff, M.J., "Supersingletons", Phys. Lett. B203 (1988) 229.

[BFH1] Binegar, B., Fronsdal, C. and Heidenreich, C., "Conformal QED", J. Math. Phys. 24 (1983) 2828.

[BFH2] Binegar, B., Fronsdal, C. and Heidenreich, C., "De Sitter QED", Ann. Phys. 149 (1983) 254. 
[BFH3] Binegar, B., Fronsdal, C. and Heidenreich, C., "Linear, conformal quantum gravity",Phys. Rev. D27 (1983) 2249.

[BMV] Brink, L., Metsaev, R.R. and Vasiliev, M.A., "How massless are massless fields in $A d S_{d}$, hep-th/0005136.

[DFKR] Duff, M., Ferrara, S., Khuri, R. R. and Rahnfeld, J, "Supersymmetry in dual string solitons", Phys. Lett. B356 (1995) 479.

[DLLP] Duff, M. Lu, J.t., Lü, I.I. and Pope, C. "Gauge dyonic strings and their global limit", Nuc. Phys. B529 (1998) 137.

[DLP] Duff, M., Lu, I. and Pope, C., "Heterotic phase transitions and singularities of the gauge dyonic string", Phys. Rev B378 (1996) 101.

[DN] Deser, S. and Nepomechie, R. I., "Gauge invariance versus masslessness in De Sitter spaces", Ann. Phys. 154 (1984) 396.

[EHW] Enright, T., Howe, R. and Wasllach, N., "A classification of unitary highest weight modules", in Representation Theory of Reductive Groups, P.C. Trombi Ed. Birkhäuser 1982.

[E] Evans, N.T., "Discrete series for the universal covering group of the $3+2$ De Sitter group", J. Math. Phys. 8 (1967) 170.

[FaFr] Fang, J. and Fronsdal, C. "Massless, half-integral spin fields on De Sitter space", Phy. Rev. D 22 (1980) 1361.

[FeFr1] Ferrara, S. and Fronsdal, C., "Conformal Maxwell theory as a singleton field theory on $A d S_{5}$, IIB three-branes and duality", Class. Quant. Grav. 15 (1998) 2153-2164. (hep-th/971223)

[FeFr2] Ferrara, S. and Fronsdal, C., "Gauge fields as composite boundary excitations", Phys. Lett. B 433 (1998) 19.

[FeFr3] Ferrara, S. and Fronsdal, C. "Gauge fields and singletons of $A d S_{2 p+1}$ ", Lett. Math. Phys. 46 (1998) 157-169. (hep-th/9806072)

[FFZ] Ferrara, S, Fronsdal, C. and Zaffaroni, A., "Supergravity on $A d S_{5}$ and $N=4$ superconformal Yang-Mills theory", Nucl. Phys. B 532 (1998) 153-162.

[FeP] Ferrara, S and Porrati, M. "AdS superalgebras and brane charges", Phys. Lett. B458 (1999) 43.

[FRS] Ferrara, S., Riccioni, F. and Sagnotti, A, "Tensor and vector multiplets in 6- 
dimensional supergravity", Nucl. Phys. B 1998 (1998) 115. (hep-th/9711059)

[FeS1] Ferrara, S. and Sokatchev, E., "Representations of $(1,0)$ and $(2.0)$ superconformal algebras in 6 dimensions, massless and short superfields", hep-th/0001178.

[FeS2] Ferrara, S. and Sokatchev, E., "Superconformal interpretation of BPS states in AdS geometry", hep-th/0005151.

[FlFr1] Flato, M. and Fronsdal, C, "One massless particle equals two Dirac singletons", Lett. Math. Phys. 2 (1978) 421.

[FlFr2] Flato, M. and Fronsdal, C., "Quantum field theory of singletons", J. Math. Phys. 22 (1981) 1100; and "The singleton dipole", Commun. Math. Phys. 108 (1987) 469.

[FlFr3] Flato, M. and Fronsdal, C., "Spontaneously generated field theories, zero center modules, colored singletons and the virtues of $N=6$ supergravity", in Essays in Supersymmetry, Reidel, 1986.

[FlFr4] Flato, M. and Fronsdal, C., "Three-dimensional singletons", Lett. Math. Phys. 20 (1990)?65.

[FFG] Flato, M., Fronsdal, C. and Gazeau, J.P., " Masslessness and lightcone propagation in De Sitter and 2+1 Minkowski space". Phys. Rev. D 33 (1986) 415.

[Fr1] Fronsdal, C., "Elementary particles in curved space IV. Massless particles," Phys. Rev. D 12 (1975) 3819.

[Fr2] Fronsdal, C., "Singletons and massless, integral spin fields on De Sitter space", Phys. Rev. D 20 (1979) 848.

[Fr3] Fronsdsal, C., "The Dirac supermultiplet", Phys. Rev. D26 (1982) 1988.

[Fr5] Fronsdal, C., "Massless particles, orthosymplectic symmetry and another type of Kaluza-Klein theory",in Essays in Supersymmetry, Reidel, 1986.

[Fr6] Fronsdal, C., "Three-dimensional singletons and two-dimensional conformal field theory", Int. J. Mod. Phys. A7 (1992) 2193, and "A model for QCD in three dimensions", in Proceedings of the Colloque Rideau, Paris 1995.

[Fr7] Fronsdal, C., "Some open problems with higher spins", Proceedings of the supergravity workshop at Stonybrook, September 1979. P. van Nieuwenhuizen and D.Z. Freedman, Ed.s. 
[FH] Fronsdal, C and Heidenreich, W., "Linear De Sitter gravity", J. Math. Phys. 28 (1987) 215.

[FN] Freedman, D. and Nikolai, H., "Multiplet shortening in $O s p(N / 4)$, Nucl. Phys. B237 (1984) 342. gravitational interaction of massless high-spin fields", Phys. Lett. 189B (1987) 89./

[GKP] Gubser, S.S., Klebanov, I.R. and Poliakov, A.M., "Gauge theory correlators from non critical string theory", Phys. Lett. B48 (1998) 105. (hep-th/9802109)

[Gu1] Gunaydin, M., "Unitary superalgeras of $O s p(1 / 32, R)$ and M-theory", Nucl. Phys. B528 (1998) 432.

[Gu2] Gunaydin, M., "AdS.CFT dualities and the unitary representations of non ccompact groups and supergroups: Wigner versus Dirac", hep-th/0005168.

[GiT] Gibbons, C.W. and Townsend, P.K., "Vacuum interpolation in supergravity via super p-branes", Phys. Rev. 71 (1993) 3754.

[GVNW] Gunaydin, M., van Nieuwenhuizen, P. and Warner, N., "General construction of the unitary representations of anti De Sitter superalgebras and the spectrum of the $S_{4}$ configuration of eleven dimensional supergravity", "Nucl. Phys. B255 (1985) 543.

[GuT] Gunaydin, M. and Takemae, S. "Unitary superalgebras of $O s p\left(8^{*} / 4\right)$ and $A d S_{7} / C F T_{6}$ duality, hep-th/9910110.

[Ha] Halyo, E., "Supergravity on $A d S(4 / 7) \times S(7 / 4)$ and M-branes", JHEP 9804,011 (1998). (hep-th/9803077)

[Ho] Horava, P. "M-theory as a holographic field theory", Phys. Rev. D 59 (1989) 046004.

[He] Heidenreich, W., Nuovo. Cim. A80 (1984) 220.

[L] Laoues, M., "Massless particles in arbitrary dimension", Math. Phys. 10 (1998) 1079.

[Mac] Mack, G., "All unitary representations of the conformal group $S U(2,2)$ with positive energy", Commun. Math. Phys. 55 (1977) 1.

[Mal] Maldacena, J., " The large $N$ limit of superconformal field theories and supergravity", Adv. Theor. Math. Phys. 2 (1998) 231. hep-th/9705104.

[Me1] Metsaev, R. R., "Arbitrary spin massless bosonic fields in $d$-dimensional anti De 
Sitter space", hep-th/9810231.

[Me2] Metsaev, R. R., "Massless mixed symmetry boson and fermion fields in anti De Sitter space", Phys. Lett. B354 (1995) 78.

[Mi] Minwalla, S., "Particles on $A d S(4 / 7)$ and primary operators of $M(2)$ brane and $M(5)$ brane world volumes", JHEP 10 (1998) 002. (hep-th/9803053)

[Mi2] Minwalla, S., "Restrictions imposed by Superconformal invariance on quantum field theories", Adv. Theor. Mat. Phys. 2 (1998) 781. (hep-th/9712074)

[N] Nahm, W., "Supersymmetries and their representations", Nucl. Phys. B 135 (1978) 149.

[NST] Nicolai, H., Sezgin, E. and Tanii, Y., "Singleton representations of $O s p(N / 4)$ ", Nucl. Phys. B305 (1988) 483.

[P] Percoco, U., "The spin $\frac{1}{2}$ singleton dipole", Lett. Math. Phys. 12 (1986) 315.

[R] Romans, L.J., "The $F(4)$ gauged supergravity in six dimensions", Nucl. Phys. B269 (1986) 691.

[Sa] Sagnotti, A., "A note on the Green-Schwarz mechanism in open string theory", Phys. Lett. 294B (1992) 196.

[Se] Seiberg, N., "Nontrivial fixed point of the renormalization group in 6 dimensions", hep-th/9609061.

[SW] Seiberg, N. and Witten, E., "Commment on string dynamics in 6 dimensions", hep-th/9603003.

[Sz] Sezgin, E., "High spin $N=8$ supergravity in $A d S_{4}$, hep-th/9903020.

[Si] Siegel, W., "All free conformal representations in all dimensions", Int. J. Mod. Phys. A 4 (1989) 2015.

[St] Strominger, A., "Open p-branes", Phys. Lett. B383 (1996) 44. (hep-th/9512059)

[T] Townsend, P. K., Nucl. Phys. (Rev. Suppl.) 68 (91998) 11. (hep-th/9708034)

[W1] Witten, E., "Anti De Sitter space and holography", Adv. Theor. Math. Phys. 2 (1998) 253. (hep-th/9802150)

[W2] Witten, E., "Some comments on string dynamics", (hep-th/9507121) 